\title{
Análisis del impacto de implementación de las normas internacionales de informaciónfinanciera (NIIF) en el sector industrial de Bogotá para PYMEs*
}

\author{
Analysis of the impact of the implementation of the International Financial Reporting Standards (IFRS) in the \\ industrial sector of Bogotá for SMEs
}

Alexander Orobio Montaño ${ }^{a}$

Universitaria Agustiniana, Colombia

alexander.orobiom@uniagustiniana.edu.co

ORCID: http://orcid.org/0000-0003-4491-4835

Edgar Manuel Rodriguez Rodriguez

Universitaria Agustiniana, Uniagustiniana, Bogotá,

Colombia, Colombia

ORCID: http://orcid.org/0000-0002-6537-0534

Juan Carlos Acosta Quevedo

Universidade Federal do Paraná, Brasil

ORCID: http://orcid.org/0000-0002-1523-3873
DOI: https://doi.org/10.11144/Javeriana.cc19-48.aiin Redalyc: http://www.redalyc.org/articulo.oa? id $=151557795008$

Fecha de recepción: 21 Mayo 2018 Fecha de aprobación: 12 Octubre 2018 Fecha de publicación: 20 Diciembre 2018

\section{Resumen:}

Este artículo trata sobre los principales impactos de la implementación de las normas internacionales de información financiera (NIIF) para pymes en el sector industrial de Bogotá. La investigación abarcó cuatro fases: capacitación, diagnóstico y utilización de recursos, elaboración de políticas contables y elaboración del estado de situación financiera de apertura. Se aplicó una encuesta a funcionarios del área contable o financiera en 403 pymes del sector industrial de la Ciudad y una entrevista semi-estructurada en profundidad a altos funcionarios del área administrativa, contable o financiera en 10 pymes del sector industrial. Los resultados revelaron la falta de compromiso desde la administración de las organizaciones, ya que es la responsable de la implementación de esta normatividad, hecho que se evidenció en la resistencia institucional para ponerla en marcha dentro de las pymes. La implementación de las NIIF en las pymes ha generado un gran impacto no solo en las áreas contables y financieras, sino en todas las áreas de las diferentes organizaciones.

Palabras clave: pymes, NIIF, información financiera, normas internacionales.

\begin{abstract}
:
The objective of this paper is to establish an analysis of the main impacts of the implementation of international financial reporting standards (IFRS) for SMEs in the industrial sector of Bogotá. The study is considered from the research bets in the University Augustinian - Uniagustiniana for the period 2016 - 2017. The quantitative analysis was determined from four phases: training, diagnosis and use of resources, preparation of accounting policies and preparation of the statement of the financial position of opening. A direct survey was applied to officials of the accounting or financial area in 403 SMEs from the industrial sector of the city and in-depth, structured interviews with senior officials in the administrative, accounting or financial area in 10 SMEs from the industrial sector. The results revealed the lack of commitment on the part of the administrations of the organizations, since they are responsible for the implementation of this regulation - a fact that was made evident in the institutional resistance to implement it within the SMEs. The implementation of the NIIF in the SMEs, has generated a great impact not only in the accounting and financial areas, but also in all areas of the different organizations.
\end{abstract}

Keywords: pymes, IFRS, financial information, international standards.

Notas de autor

${ }^{a}$ Autor de correspondencia. Correo electrónico: alexander.orobiom@uniagustiniana.edu.co 


\section{Introducción}

El gobierno de Colombia, desde el año 2009 ha querido que el país homologue las normas en materia contable a nivel internacional, es por ello que emitió la Ley 1314 en 2009 y la reglamento a través de decretos entre los años 2012 a 2016. Este asunto de implementación de normas internacionales separa tres grupos; las pymes se encuentran en el segundo. La revista Dinero en su publicación "Llego la hora de que las pymes se pasen a las NIIF" resalta que una empresa que aplique NIIF puede ser más atractiva para inversionistas extranjeros y tener beneficios en procesos de compra o alianzas. Igualmente, destaca que para las pymes es más exigente el proceso de aprendizaje y la aplicación a las prácticas, pues, son empresas con menos infraestructura y cuentan con carencia de asesoría en este proceso.

El análisis del impacto de la implementación de las NIIF busca diagnosticar cómo las pymes del sector industrial de Bogotá han enfrentado la consumación de esta norma en la figura de obligatorio cumplimiento dentro del marco legal contable y financiero en Colombia; pero también la relevancia que las empresas le otorgan a la convergencia a las NIIF para facilitar el ingreso de sus productos y /o servicio en la dinámica de los mercados internacionales. La seguridad de la información financiera y /o contable es un tema que ha venido ganando relevancia por la certeza que esta debe ofrecer para la toma de decisiones con el menor grado de incertidumbre financiero para las organizaciones; también para ofrecer información pertinente que les permita a los organismos de control monitorear el comportamiento financiero de las pymes y ofrecer así seguridad y estabilidad financiera a los sistemas.

El artículo se plantea en cinco secciones, a saber, en primer lugar, se expone la revisión de la literatura y los antecedentes; en segundo lugar se presenta el marco teórico de las NIIF; en tercer lugar se presenta el marco metodológico y el protocolo estadístico para la determinación de la muestra a la cual se le aplicó la encuesta y las entrevistas, como instrumentos de levantamiento de la información; la cuarta sección se dedica a los resultados del estudio y finalmente se presentan las reflexiones a manera de conclusiones.

\section{Revisión de la literatura}

Las Normas Internacionales de Información Financiera (NIIF), como conjunto de parámetros expedidos por la IASB (International Accounting Standards Board) emergen como consecuencia de la globalización económica y la dinámica de los mercados internacionales. Con la implementación de las NIIF en Colombia se busca contar con datos de mayor calidad y transparencia en la disciplina contable y financiera. Como punto de partida de esta indagación se presentan algunas posturas sobre el tema de implementación de las NIIF en las empresas, específicamente en las pymes, y los impactos de ejecución.

Macías y Quintero (2016) hicieron un análisis de la literatura sobre la implementación de IFRS en países con menor nivel de desarrollo, de África, América Latina, Asia y Europa Oriental. Los autores clasificaron los casos en dos grupos: por un lado, los trabajos que abordan las experiencias de más de 30 países en desarrollo, y por otro lado, los estudios de caso por país, donde se analizan tanto la evolución histórica de los estándares contables aplicados en el país, como las características del proceso de adopción de IFRS y los impactos del proceso.

El estudio de Abrego, Sánchez y Medina (2016) muestra que durante los últimos años los sistemas de información han constituido uno de los principales ámbitos de estudio en el área de organización de empresas, ocasionado por la necesidad de identificar su valor empresarial. Los autores desarrollan un modelo de evaluación del éxito de los sistemas de información para las pymes, con el objetivo de determinar su influencia en los resultados organizacionales, con información de 133 empresas del estado de Tamaulipas, México. Los resultados obtenidos permiten deducir que las empresas que se preocupan más por mejorar la calidad del sistema, la calidad de la información y la del servicio informático, favorecen sus resultados organizacionales. 
Salazar (2013) considera que la aplicación de nuevas normas contables en las entidades, supone costos y beneficios; los diversos efectos de la aplicación de las NIIF en entidades que participan de los mercados de capitales. Con base en la experiencia de un estudio de caso en el cual se implementó las NIIF en una mediana empresa colombiana, evidenció mejoras en la calidad de la información contable, al tiempo que caracteriza las dificultades que pueden experimentar las pymes en este proceso.

Parra (2016) considera que las NIIF han constituido un reto para el entorno empresarial de las pymes, en particular para el nivel directivo. En algunas empresas la implementación de las NIIF depende del nivel académico que posea el gerente o el contador. El impacto causado por la implementación de las normas internacionales se resume en un incremento de la transparencia de la información financiera y en una afectación a las cuentas del estado situacional financiero.

Cañibano y Gisbert (2007) caracterizan una etapa de cambio a escala mundial, hacia el fortalecimiento de la calidad y la armonización de la información financiera, suministrada en los mercados de capitales de todo el mundo. Uno de los organismos que lidera este proceso es el IASB, que ha recibido el reconocimiento y el apoyo internacional como organismo emisor de una normativa contable internacional de calidad; los vínculos con entidades como la Organización Internacional de Comisiones de Valores (IOSCO por sus siglas en inglés), la Comunidad Europea y otros entes reguladores de impacto en la definición de estándares contables.

Salas (2015) hace un importante aporte a través del análisis de la información financiera del año 2014 correspondiente a la Cooperativa Coopactiva, entidad que fue seleccionada con el propósito de dar a conocer la dinámica contable del proceso de convergencia y los impactos financieros, como resultado de la aplicación hacia los estándares internacionales de Contabilidad e información financiera, de conformidad con la Ley 1314 del 2009 y su decreto reglamentario 3022 de 2013, NIIF Para pymes aplicables al sector Solidario en Colombia.

Rodríguez (2016) presenta una revisión bibliográfica sobre la gestión financiera en las pymes a través de Google Académico y Scopus, analiza 137 referencias de las cuales tuvo en cuenta 17 relacionadas directamente con el concepto de gestión financiera como herramienta fundamental para la administración, planificación y control de los recursos financieros de las pymes. Determinó que la gestión financiera en pymes está caracterizada por insolvencia y poca liquidez como producto de políticas financieras poco efectivas o por el desconocimiento del propietario-gerente, con respecto al manejo adecuado de sus recursos.

Muñoz (2016) desarrolla un estudio de caso basado en Ferracol S.A.S., empresa dedicada a la comercialización de productos de ferretería. El autor analiza el impacto de la implementación de las NIIF en el rubro activos fijos y en el segmento tributario el rubro de patrimonio, dado su importancia dentro de la estructura del balance en este tipo de empresas.

Por todo lo anterior, se concluye que las investigaciones destacan la importancia de la implementación de las NIIF en las pymes para alcanzar un óptimo desarrollo y los resultados esperados en el ámbito contable y financiero, identificando así el impacto que genera dicha implementación, además por las inversiones y los esfuerzos que debe disponer las organizaciones.

\section{Marco histórico de las NIIF}

En Estados Unidos la Financial Accounting Standards Board - FASB. (Junta de Normas de Contabilidad Financiera), fundada en 1973, reemplazó a la Comisión de principios de contabilidad que venía operando desde 1959 y cuya función principal era crear normas de contabilidad, así como emitir pronunciamientos en materia contable que guían la forma de presentar la información financiera en Estados Unidos del sector privado independiente, sin fines de lucro. 
TABLA 1

Historia de las normas contables en Estados Unidos

\begin{tabular}{|c|c|c|}
\hline Año & Entidad & Función \\
\hline 1957 & $\begin{array}{l}\text { Instituto Americano de } \\
\text { Contadores Públicos }\end{array}$ & $\begin{array}{l}\text { Fundado desde } 1987 \text { como Asociación Americana de } \\
\text { Contadores Púbicos (AAPA). Su misión es alimentar el } \\
\text { éxito del negocio global, contadores, proporcionando los } \\
\text { conocimientos, los recursos y la defensa más relevantes y } \\
\text { proteger el interés público en evolución. }\end{array}$ \\
\hline 1972 & $\begin{array}{l}\text { Fundación de Contabilidad } \\
\text { Financiera }\end{array}$ & $\begin{array}{l}\text { Apoya y supervisa el FASF. Establece las normas contables, } \\
\text { estas son las reglas que determinan la forma que el lenguaje } \\
\text { está escrito. Estas normas se conocen colectivamente como } \\
\text { los principios generalmente aceptados de Estados Unidos o } \\
\text { contabilidad USGAAP. }\end{array}$ \\
\hline 1973 & $\begin{array}{l}\text { Consejo de Normas de } \\
\text { Contabilidad Financiera }\end{array}$ & $\begin{array}{l}\text { Reemplazola comisión de principios de contabilidad, que } \\
\text { inicio desde } 1959 \text {. Establece la contabilidad financiera e } \\
\text { información financiera para empresas privadas con y sin } \\
\text { fines de lucropara organizaciones que siguen principios de } \\
\text { contabilidad generalmente aceptados (PCGA) }\end{array}$ \\
\hline 1977 & $\begin{array}{l}\text { Federación Internacional de } \\
\text { Contadores }\end{array}$ & $\begin{array}{l}\text { Su función es servir al interés público mediante el } \\
\text { fortalecimiento de la profesión y la contribución al } \\
\text { desarrollo de fuertes economías intemacionales. }\end{array}$ \\
\hline 1984 & $\begin{array}{l}\text { Junta de Normas de } \\
\text { Contabilidad } \\
\text { Gubermamentales }\end{array}$ & $\begin{array}{l}\text { Desarrolla y emite las normas de contabilidad a través de un } \\
\text { proceso de transparente e inclusivo, con la intención de } \\
\text { promover la información financiera que proporcione } \\
\text { información útil para los contribuyentes, funcionarios } \\
\text { públicos los inversores y otras personas que utilizan los } \\
\text { informes financieros. }\end{array}$ \\
\hline
\end{tabular}

Los Principios de Contabilidad Generalmente Aceptados (GAAP por sus siglas en inglés) son las normas contables que forman la base del sistema de información financiera de los Estados Unidos. Son establecidos y mantenidos por un organismo de normalización independiente, el Financial Accounting Standards Board (FASB). Aunque los US GAAP continúan sirviendo bien a los intereses de los inversionistas y otras partes interesadas, no disminuye la necesidad - en los Estados Unidos y en el extranjero - de seguir trabajando arduamente para apoyar el desarrollo de estándares de contabilidad aceptados a nivel mundial y de alta calidad. (White, 2017)

En la figura 1 se presenta una síntesis de las normas a nivel internacional.

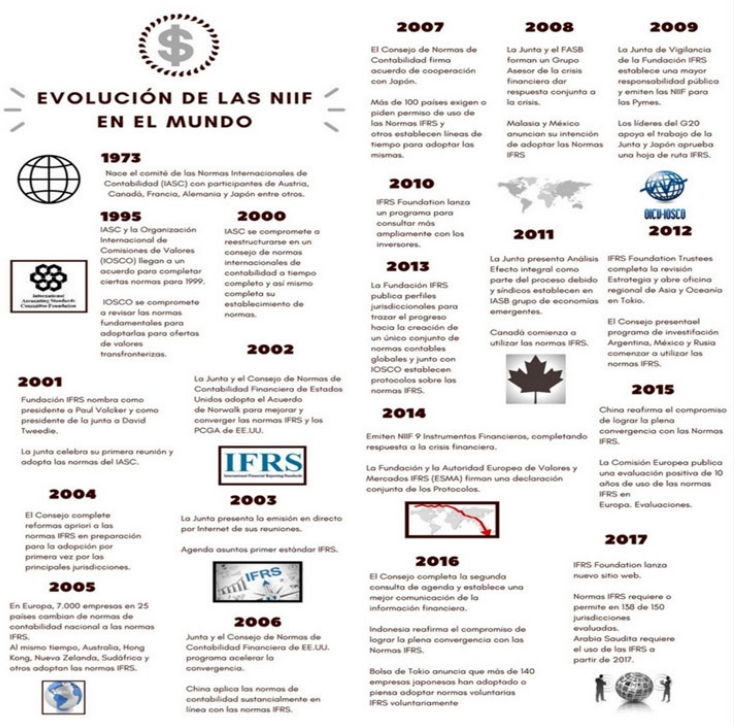

FIGURA 1. Historia de las NIIF 
Por otro lado, el International Financial Reporting Standards Foundation (IFRS) es una organización sin ánimo de lucro, organización de interés público establecido para desarrollar un conjunto único de alta calidad, comprensible y aplicable a las normas contables internacionales aceptadas a nivel mundial en estándares y para promover y facilitar la adopción de los estándares.

Según lo establecido por la IFRS Foundation (2016), las NIIF para pymes fueron emitidas por el IASB, Junta Internacional de Normas de Contabilidad, en julio de 2009; estas entraron en vigencia el 1 de enero de 2017 y comprenden la parte A que contiene los requerimientos y la parte B que contiene los documentos complementarios y comprende 35 secciones.

TABLA 2

Listado de las NIIF para pymes

\begin{tabular}{ll}
\hline Sección & Nombre de la sección \\
\hline 1 & Pequeñas y medianas entidades \\
2 & Conceptos y principios generales \\
3 & Presentación de estados financieros \\
4 & Estado de situación financiera \\
5 & Estado del resultado integral y estado de resultados \\
6 & Estado de cambios en el patrimonio y estado de resultados y ganancias acumuladas \\
7 & Estado de flujos de efectivo \\
8 & Notas a los estados financieros \\
9 & Estados financieros consolidados y separados \\
10 & Políticas contables, estimaciones y errores \\
11 & Instrumentos financieros básicos \\
12 & Otros temas relacionados con los instrumentos financieros \\
13 & Inventarios \\
14 & Inversiones en asociadas \\
15 & Inversiones en negocios conjuntos \\
16 & Propiedades de inversión \\
17 & Propiedades, planta y equipo \\
18 & Activos intangibles distintos de la plusvalia \\
19 & Combinaciones de negocio y plusvalía \\
20 & Arrendamientos \\
21 & Provisiones y contingencias \\
22 & Pasivos y patrimonio \\
23 & Ingresos de actividades ordinarias \\
24 & Subvenciones del gobierno \\
25 & Costos por préstamos \\
26 & Pagos basados en acciones \\
27 & Deterioro del valor delos activos \\
28 & Beneficios a los empleados \\
29 & Impuesto a las ganancias \\
30 & Conversión dela moneda extranjera \\
31 & Hiperinflación \\
32 & Hechos ocurridos después del periodo sobre el que se informa \\
33 & Informaciones para revelar sobre partes relacionadas \\
34 & Actividades especiales \\
35 & Transición a la NIIF para las pymes \\
\hline
\end{tabular}

Fuente: IFRS Foundation (2016)

Según la International Standards of Accounting and Reporting -ISAR- (2017), fue Fundada en 1982 por las Naciones Unidas Consejo Económico y Social (ECOSOC), a través de su División de Inversión 
y Empresa. La ISAR es el grupo de trabajo intergubernamental dedicado a la transparencia empresarial y problemas de contabilidad a nivel corporativo, aborda una variedad de temas en negocios de contabilidad e informes a fin de mejorar la comparabilidad global y fiabilidad de los informes corporativos, presta asistencia a los países en desarrollo y a las economías en transición, en la aplicación de las mejores prácticas en materia financiera y gobierno corporativo, con el interés de mejorar el clima de inversión y promover el desarrollo sostenible mediante un proceso integrado de investigación, creación de consenso intergubernamental, difusión de información y cooperación técnica.

TABLA 3

Normas internacionales en Latinoamérica- Reportes ROSC-FMI (2008)

\begin{tabular}{ll}
\hline País & Fecha \\
\hline Chile & Julio de 2001 y septiembre de 2007 \\
Colombia & Octubre de 2006 \\
Costa Rica & Agosto de 2002 \\
República Dominicana & Junio de 2006 \\
Ecuador & Marzo de 2003 \\
El salvador & Diciembre de 2004 \\
Guatemala & Junio de 2005 \\
Honduras & Julio de 2005 \\
México & Junio de 2003 \\
Nicaragua & Diciembre de 2005 \\
Panamá & Octubre de 2006 \\
Paraguay & Junio de 2006 \\
Perú & Octubre de 2003 \\
Uruguay & Octubre de 2001 \\
\hline
\end{tabular}

Fuente: Mejía, Montes y Montilla (2008).

Con respecto a los Reportes de Observancia de Aplicación de Códigos y Estándares -ROSC-, Mejía, Montes, Montilla (2008) muestra que el Banco Mundial y el Fondo Monetario Internacional desarrollaron varios estudios para determinar la calidad de los sistemas contables en Latinoamérica; los cuales se convirtieron en un referente importante para que las partes puedan evaluar la experiencia en el proceso de adopción, y también para que los países que se encuentran en proceso de adopción, estandarización, o en proyecto de realizarlo tengan un referente que les permita recorrer el camino con mayor facilidad. 
TABLA 4

Marco Legal Colombiano. Implementación de las NIIF

\begin{tabular}{|c|c|c|}
\hline Grupo 1 NIIF plenas & Grupo 2 NIIF para pymes & Grupo 3 Microempresas \\
\hline Ley 1314 de 2009 & Ley 1314 de 2009 & Ley 1314 de 2009 \\
\hline Decreto 2784 de 2012 & & Decreto 2706 de 2012 \\
\hline Decreto 1851 de 2013 & Decreto 3022 de 2013 & Decreto 3019 de 2013 \\
\hline \multicolumn{3}{|l|}{ Decreto 3023 de 2013} \\
\hline \multicolumn{3}{|l|}{ Decreto 3024 de 2013} \\
\hline Decreto 2267 de 2014 & Decreto 2129 de 2014 & \\
\hline \multicolumn{3}{|l|}{ Decreto 2615 de 2014} \\
\hline Decreto Unico Reglamentario & Decreto Unico Reglamentario & Decreto Unico Reglamentario \\
\hline 2420 de 2015 & 2420 de 2015 & 2420 de 2015 \\
\hline Anexo: D.R 2615 de 2014 y DR & Anexo D.R 3022 de 2013 y DR & Anexo DR 2706 de 2012 \\
\hline 2131 de 2016 & 2131 de 2016 & \\
\hline $\begin{array}{l}\text { Decreto Reglamentario } 2496 \text { de } \\
2015\end{array}$ & $\begin{array}{l}\text { Decreto Reglamentario } 2496 \text { de } \\
2015\end{array}$ & $\begin{array}{l}\text { Decreto Reglamentario } 2496 \text { de } \\
2015\end{array}$ \\
\hline
\end{tabular}

Fuente: información tomada de Principales Impacto de la aplicación de las ISAE.

En Colombia el Gobierno Nacional, a través del Congreso de la República y en cabeza de los Ministerios de Industria, Comercio y Turismo, de Hacienda y Crédito Público, promulgó la Ley 1314 de 2009 y ha reglamentado esta ley con unos decretos desde el año 2012 hasta el 2016, con el fin de garantizar la implementación de las Normas Internacionales de Información Financiera.

A mediados de julio de 2009, el Congreso de Colombia emitió la Ley 1314, para regular los principios y normas de contabilidad e información financiera y las Normas de Aseguramiento de Información para aplicar en Colombia. Esta ley también señaló las autoridades competentes, el procedimiento para su expedición y determinó las entidades responsables de vigilar su cumplimiento. Posteriormente, el Consejo Técnico de la Contaduría Pública presentó la dirección estratégica relacionada con el proceso de convergencia de las mencionadas normas en lineamiento con los estándares internacionales y estableció tres grupos de usuarios, para iniciar la convergencia de forma gradual. La Superintendencia Financiera de Colombia acogió la dirección del Consejo Técnico respecto a los plazos y fechas de inicio para la convergencia de las normas colombianas a las internacionales de información financiera.

La Dirección de Gestión de Fiscalización de la DIAN en su documento incidencia fiscal en el periodo de transición de las NIIF del II conversatorio con contadores publicado el 25 de noviembre de 2015, presenta el marco normativo de las NIIF para el sector privado así:

GRUPO 1: Decreto 2784 de 2012 (DR 1851, 3023, 3024 de 2013 y 2267 de 2014) Aplican NIIF Plenas

GRUPO 2 Decreto 3022 de 2013 (DR 2129, 2267 de 2014) Aplican NIIF pymes

GRUPO 3 Decreto 2706 de 2012 (DR 3019 de 2013) Aplican Anexo Decreto

Con respecto a las pymes, el documento de Orientación Técnica No. 005 sobre aplicación de las NIIF para las pymes, 9 de noviembre de 2015. Consejo Técnico de la Contaduría Pública, presenta el cronograma de adopción por primera vez (Estado de Situación Financiera de Apertura - ESFA) de la siguiente forma:

En el ejercicio de 2015:

1 de enero de 2015: Fecha de transición hacia la NIIF pymes

31 de diciembre de 2015: última vez que se informa sobre pcga y primer periodo de información NIIF pymes.

En el ejercicio de 2016 :

31 de diciembre de 2016: primeros estados financieros NIIF pymes con información comparativa.

En el ejercicio de 2017: 
31 de diciembre de 2017: Información recurrente conforme NIIF pymes.

El 4 de septiembre de 2017, la Superintendencia de Sociedades realizó en la ciudad de Bogotá el III Encuentro Nacional de Construcción Conjunta y con respecto a la recepción de información financiera consolidada a 31 de diciembre de 2017, presenta como sociedades requeridas al 26 de julio de 2017, un total de 25.903 de las cuales pertenecen a las empresas del grupo dos, 22.326 empresas, las empresas grupo 1 más voluntarias presentan 3.577 empresas, para un total de 25.903 empresas requeridas en el año 2017.

De acuerdo con lo anterior se puede deducir que del total de sociedades requeridas en el año 2017 por la Superintendencia de Sociedades, el 86\% de las empresas corresponden al grupo 2. Con corte al 26 de julio de 2017 estas empresas del grupo 2 debieron enviar su información financiera con corte a 31 de diciembre de 2016, del cual se obtuvo la siguiente recepción: 14.246 empresas enviaron a tiempo la información $(63,8 \%) ; 4.443$ empresas enviaron la información de forma extemporánea (19,9\%), mientras 3.637 empresas no enviaron la información (16,3\%).

\section{Metodología}

En Bogotá existen 704.012 empresas, de las cuales 16.625 son pequeñas empresas y 3.721 empresas de tamaño mediano; con base en este criterio las organizaciones con mayor número de empresas son las denominadas pymes, las cuales gozan de un conjunto de beneficios que pueden ser obtenidos por sus fundadores desde su creación (Cámara de Comercio de Bogotá, 2017).

Para el presente estudio se determinó una muestra de 403 pymes del sector industrial, que posibilitaron aplicar 403 encuestas parciales y directas y 10 entrevistas semi-estructuradas en profundidad al personal del área financiera y/o contable. El análisis cuantitativo se abordó desde las consideraciones de Martínez (2012) utilizando Excel como herramienta tecnológica para el tratamiento de los datos. Dada la población de las pymes del sector industrial de Bogotá, se procedió a determinar la muestra estadística para el presente estudio; no sin antes caracterizar el universo de las pymes del sector industrial por localidades, como está organizada políticamente la Ciudad. Así se muestra en la tabla 5. 
TABLA 5

Distribución pymes industriales por localidad

\begin{tabular}{|l|r|r|r|}
\hline \multicolumn{5}{|c|}{ Clasificación } \\
\hline \multicolumn{1}{|c|}{ Localidad } & Pequeñas & Medianas & Total General \\
\hline Antonio Nariño & 162 & 20 & 182 \\
\hline Barrios Unidos & 443 & 63 & 506 \\
\hline Bosa & 50 & 11 & 61 \\
\hline Candelaria & 18 & 2 & 20 \\
\hline Chapinero & 218 & 36 & 254 \\
\hline Ciudad Bolívar & 29 & 15 & 44 \\
\hline Engativá & 475 & 87 & 562 \\
\hline Fontibón & 338 & 156 & 494 \\
\hline Kennedy & 551 & 78 & 629 \\
\hline Los Mártires & 369 & 72 & 441 \\
\hline Puente Aranda & 693 & 213 & 906 \\
\hline Rafael Uribe & 131 & 11 & 142 \\
\hline San Cristóbal & 31 & 4 & 35 \\
\hline Santa Fe & 86 & 15 & 101 \\
\hline Suba & 252 & 36 & 288 \\
\hline Teusaquillo & 120 & 22 & 142 \\
\hline Tunjuelito & 74 & 8 & 82 \\
\hline Usaquén & 271 & 59 & 330 \\
\hline Usme & 5 & 0 & 5 \\
\hline Total general & 4316 & 908 & 5224 \\
\hline
\end{tabular}

Fuente: Cámara Comercio, 2017.

Con esto llegamos a otro dato que debe indicarse para la elaboración de la muestra, esto es la población finita, siendo para el caso, usados los datos previamente expuestos sobre número de pymes en la ciudad, donde se da cuenta de 20.346 establecimientos de este tipo; dado que esta es una cifra global de la ciudad y no está por localidad, la muestra que a continuación se calcula es aleatoria sistemática y no estratificada por las condiciones de diferenciación que se plantean en Martínez (2012). Con las anteriores indicaciones, se procede a determinar la muestra siguiendo la siguiente ecuación: $\mathrm{n}=\frac{N * K_{a}^{2} * p * q}{N_{*}^{2}+K_{a}^{2} * p * q}$

Dónde:

$n=$ Muestra o número de Encuestas

${ }_{N}=$ Población

$K_{\alpha}=$ Valor de la tabla Normal para un nivel de significancia

$p=$ Probabilidad de éxito

$q=$ Probabilidad de fracaso

$\epsilon=$ Error absoluto de la muestra

Población: $\mathrm{N}=2000$

Intervalo de confianza: $95,5 \% \rightarrow \mathrm{Z}=2$

Error admisible: $4,5 \% \rightarrow \mathrm{E}=0,045$

Varianza máxima: $\mathrm{P}=0,5 \cdot \mathrm{Q}=0,5$.

$$
\mathrm{No}=\frac{z^{2} P Q}{E^{2}}=\frac{(2)^{2}(0,5)(0,5)}{(0,045)^{2}}=493,82
$$

Corrección del tamaño de la muestra. 


$$
\mathrm{N}=\frac{n_{0}}{1+n_{N}^{n_{0}}}=\frac{493,82}{1+\frac{493,82}{2000}}=396 \approx 400
$$

Teniendo en cuenta los resultados obtenidos y el nivel de confianza y error que se deseen trabajar para efectos del presente estudio con un nivel de confianza de $95 \%$ y un error del $5 \%$, se realizaron 358 encuestas a empresas pymes del sector industrial de la ciudad de Bogotá.

\section{Resultados}

El análisis de la implementación de las NIIF para las pymes del sector industrial de Bogotá se abordó con 403 unidades empresariales, en las cuales se aplicó una encuesta directa y presencial sobre la experiencia en la implementación de esta norma internacional y una entrevista semi-estructurada en profundidad, a 10 altos directivos del área administrativa, financiera y/o contable de pymes. De la muestra el $81 \%$ son microempresas, el $18 \%$ pequeñas empresas y el $1 \%$, medianas empresas.

En el proceso de levantamiento de información sobre la implantación de las NIIF en las pymes del sector industrial se consideró importante determinar el nivel de formación académica de los informantes; la formación académica de las personas que respondieron la encuesta, muestra que el $75 \%$ tienen estudios profesionales y el 25\% no supera el nivel de escolaridad básica.

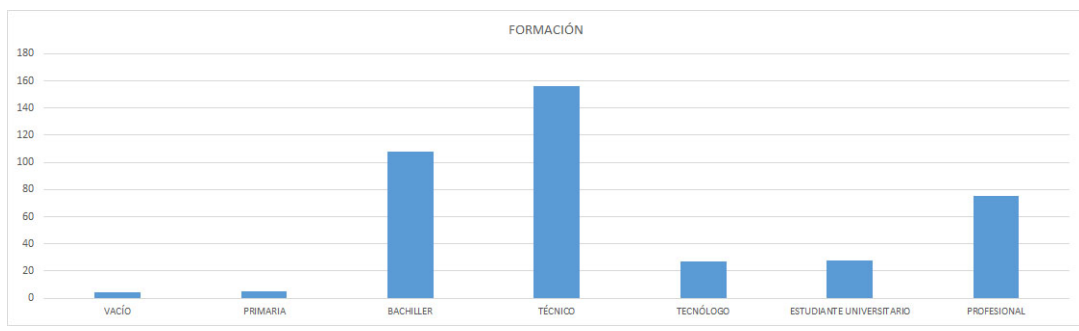

FIGURA 2. Formación académica de los informantes

Fuente: elaboración propia.

Otro aspecto que se consideró importante fue conocer el tiempo de vinculación laboral del empleado en la pyme, buscando que ellos tuvieran conocimiento sobre el proceso de implementación de la norma; en consecuencia, el tiempo de vinculación laboral de los encuestados en la empresa es en promedio de 23,5 meses, el 50\% no supera los 18,4 meses de vinculación. Ver figura 3.

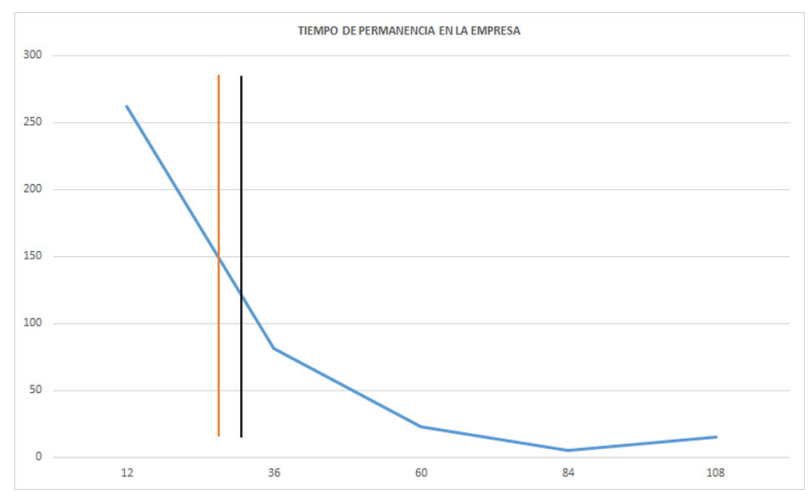

FIGURA 3. Tiempo de permanencia de los informantes en la empresa

Fuente: elaboración propia.

Enfatizando en el proceso de implementación de las NIIF, los instrumentos se aplicaron con base en cuatro categorías fundamentales como son:

1. El enfoque de conocimiento general de las NIIF

2. El enfoque de fase de capacitación 
3. El enfoque de fase diagnóstico, elaboración de políticas y ESFA

4. El enfoque de implementación general de las NIIF

Estas categorías mediaron la búsqueda de la caracterización del proceso de implementación de las pymes, y por supuesto el diagnóstico o la intensión de medir el impacto. En esta etapa los encuestados califican, entre 1 (Muy difícil) y 5 (Muy fácil), su desempeño y proceso de crecimiento personal con una valoración promedio de 3,5; la gran mayoría valoró su crecimiento con 4, pero es de anotar que el $25 \%$ lo valoró con una calificación inferior a 3 . Ver figura 4.

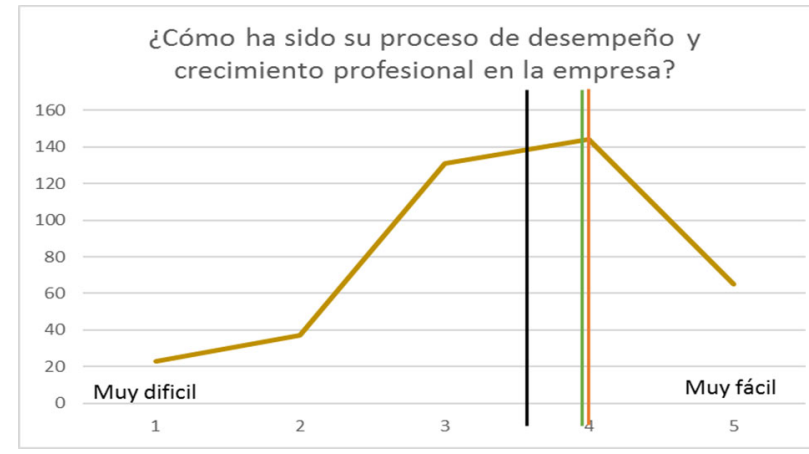

FIGURA 4. Crecimiento y desarrollo profesional de los informantes en la pyme

Fuente: elaboración propia.

Las expectativas dentro de la empresa muestran el compromiso de al menos el $60 \%$ de los encuestados que se conciben con un ascenso y participando del crecimiento de esta. El 20\% espera un crecimiento personal, independientemente de su situación con la empresa y sólo el $14 \%$ esperan la oportunidad para terminar su vinculación con la misma. Ver figura 5.

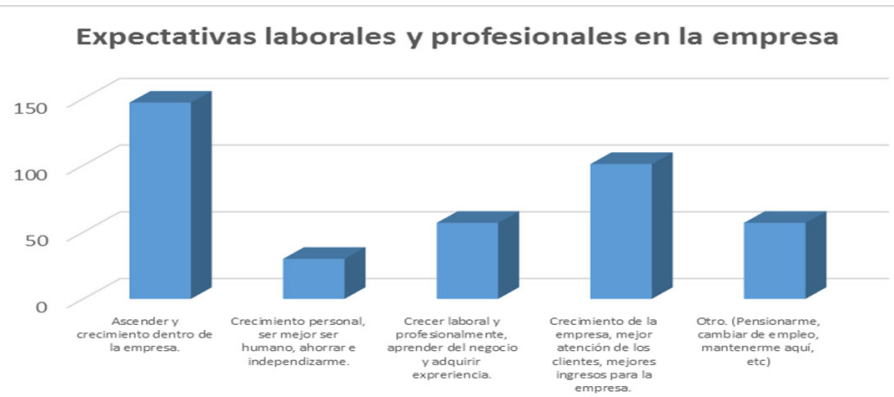

FIGURA 5. Expectativas laborales y profesionales de los informantes en la pyme Fuente: elaboración propia.

Respecto al conocimiento que cada informante tiene de las NIIF se encontró que sólo el 15,6\% expresan seguridad respecto del tema. El 61\% expresan que no conocen dichas normas, y el 23,3\% manifestaron que las conocen muy poco.

En esta búsqueda se les preguntó a los informantes: “¿Conoce las partes obligatorias de las Normas Internacionales de Información Financiera de implementación de las NIIF para pymes?”; “¿Conoce la Ley 1314 de 2009, los decretos que la reglamentan y las Circulares externas 115 y 200 de la Superintendencia de sociedades?” Y “¿Conoce usted el Cronograma de aplicación del marco técnico normativo para los preparadores de información financiera para las pymes?". Se ratifica que hay una tendencia marcada hacia el desconocimiento de las NIIF en todos los aspectos señalados, con una tendencia marcada hacia el rechazo al cambio que puede producir su implementación. Por esto consideramos que la etapa de capacitación de las NIIF es considerada como fundamental. El nivel de capacitación que consideran los encuestados que tienen los encargados de la implementación de las NIIF en las empresas, se valora con un promedio de 3,1 en una escala entre 1 (Nada capacitados) y 5 (Muy capacitados); cabe resaltar que el 30\% lo valora con una nota entre 1 y 2 . 


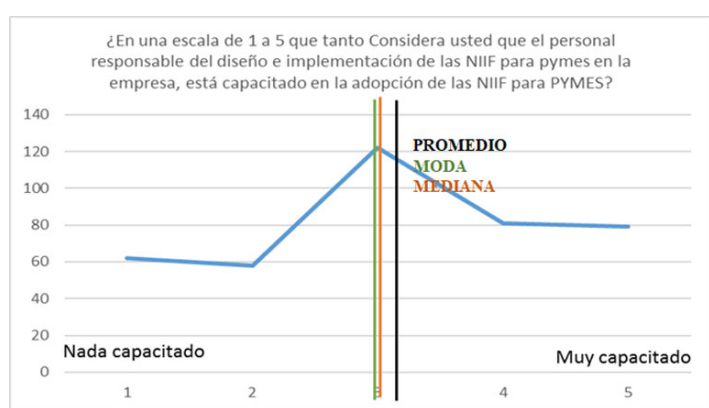

FIGURA 6. Nivel de capacitación de los responsables de implementación de las NIIF en las pyme

Fuente: elaboración propia.

Con respecto al nivel de capacitación de todo el personal de la empresa en el conocimiento de las NIIF, los informantes valoraron con una calificación promedio de 2,7 en la misma escala de la pregunta anterior; subiendo hasta el $40 \%$ el porcentaje de encuestados que valoran entre 1 y 2 , lo que resulta un nivel muy bajo de capacitación.

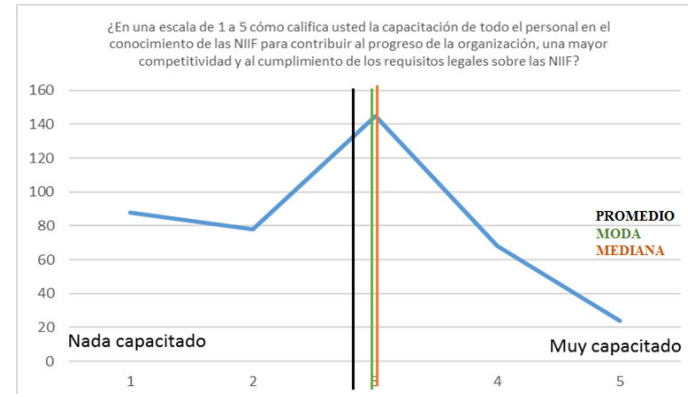

FIGURA 7. Capacitación del personal de la empresa en el tema de NIIF

Fuente: elaboración propia.

Por otro lado, la pertinencia de la inversión en capacitaciones sobre NIIF para el personal, entendido como la apertura de la empresa a nuevos mercados nacionales e internacionales, el $88 \%$ de los encuestados respondieron que es positiva y bien vista; un grupo nada despreciable consideran que no es pertinente, el $10 \%$. Sólo el $2 \%$, consideran no tener suficiente información como para emitir un juicio en este tema. Por otro lado, la inversión en tecnologías a fin de sistematizar la contabilidad en general de la empresa, fue valorada con un promedio de 4 en una escala entre 1 (Poco importante) y 5 (Muy importante); es de resaltar que se mantiene la tendencia de dar menor importancia a estos temas en las catalogadas como micro y pequeña empresa.

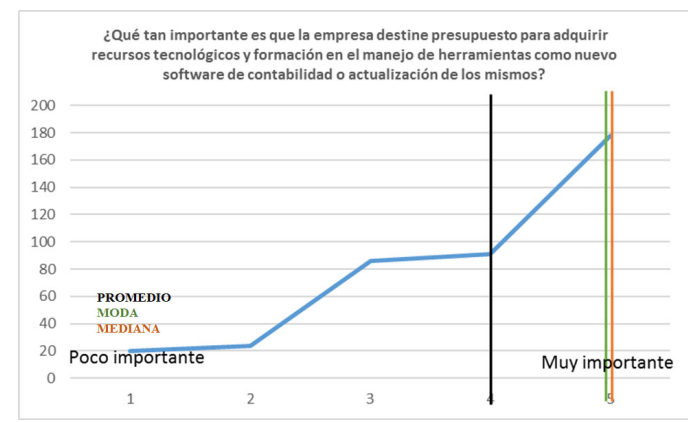

FIGURA 8. Importancia de destinación de presupuesto de tecnología de software contable

Fuente: elaboración propia.

Un comportamiento similar al de la respuesta a la pregunta anterior, se vio en la reacción a la pregunta: "¿Qué tan importante es que la empresa cuente con asesores expertos en las normas internacionales de información financiera, para acompañar y aconsejar el personal responsable de implementación y gestión de las NIIF?"; la valoración promedio fue de 4,1 aunque sólo el $21 \%$ afirma y confiere una calificación entre 1 y 2. 


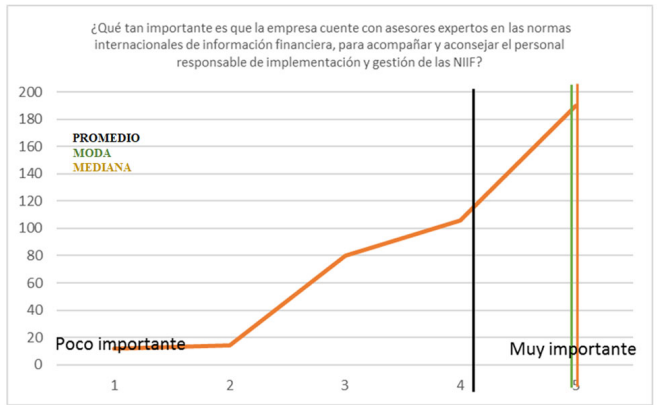

FIGURA 9. Importancia de contar en la empresa con asesores expertos en NIIF

Fuente: elaboración propia.

Enfoque fase diagnóstico, elaboración de políticas y ESFA. La sección 10 de NIIF para pymes, establece que las políticas contables son: "los principios, bases, convenciones, reglas y procedimientos específicos adoptados por una entidad al preparar y presentar los estados financieros".

Cuando se preguntó por la existencia de un manual de políticas sobre NIIF establecido formalmente en las empresas, la respuesta del 62,8\% fue que no cuentan con él, siendo consecuente con las tendencias acerca del conocimiento sobre el tema hasta el momento. Sobre la posibilidad inminente de afrontar cambios venideros en las políticas contables, como consecuencia de los nuevos estándares internacionales de contabilidad y la consideración que la empresa ha hecho de esta, se evidencia que sólo un 38,5\% lo ha contemplado y que dado este caso se tomaría por sorpresa a la mayoría, representada en un $61,5 \%$.

En cuanto al impacto que ha tenido la estructuración de la información financiera y el mejoramiento de su calidad por causa de los cambios contables debidos al proceso de implementación de las NIIF, se obtuvo una permuta que discrepa de las tendencias hasta el momento. El 73\% indicó que sí contribuyeron, aunque en la respuesta anterior se indicó que el $63 \%$ de las empresas no cuentan con un manual de implementación de NIIF.

Sobre la realización de un diagnóstico financiero actual de la compañía, se encuentra que el 44\% de los encuestados lo tienen vigente con un año de realización, mientras que el 33\% nunca lo han realizado, el restante $23 \%$ lo tiene desactualizado con una realización superior a los 5 años.

Para el cuestionamiento acerca de la existencia de un manual de políticas financieras, el $34 \%$ nunca lo ha elaborado, el 37\% lo tiene desactualizado con un tiempo superior a los 2 años, mientras que sólo el 29\% lo elaboró durante el último año.

En un $35,5 \%$ los encuestados han realizado el estado de situación de apertura durante el último año, mientras que el $28,9 \%$ lo presentó con tiempo superior a 2 años y el $35,6 \%$ no lo ha presentado nunca.

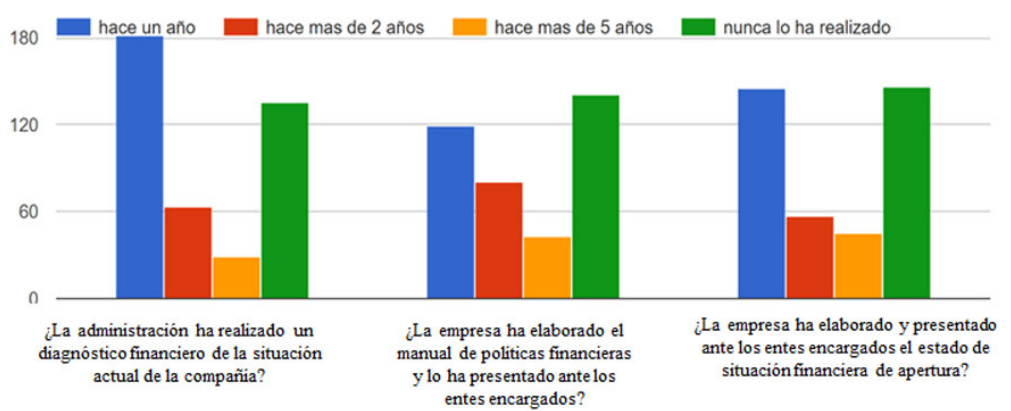

FIGURA 10. Diagnóstico sobre el proceso de implementación actual de las NIIF

Fuente: elaboración propia.

Enfoque implementación general de las NIIF. El párrafo 35.4 de la sección 35 de NIIF para pymes, requiere que la entidad que adopta por primera vez la NIIF para las pymes, debe presentar una declaración explícita y sin reservas de cumplimiento en su primer conjunto completo anual de estados. La aplicación de la Sección 35 implica cuatro pasos: 1) Determinar si una entidad es una entidad que adopta por primera vez la NIIF para las pymes; 2) Identificar la fecha de transición a las NIIF para las pymes; 3) Elaborar el estado de situación 
financiera de apertura a su fecha de transición a la NIIF para las pymes; y 4) Preparar los estados financieros que cumplen con las NIIF para las pymes, incluida la información a revelar para explicar el efecto de la transición del marco de información financiera anterior a las NIIF para las pymes.

A la pregunta: “¿Cree usted que el éxito competitivo de la entidad a nivel nacional e internacional está en el proceso satisfactorio de implementación de la normatividad internacional?" los encuestados respondieron con una valoración promedio de 3,4 en una escala que va desde 1 (Poco de acuerdo) y 5 (Muy de acuerdo); la valoración promedio y de la gran variabilidad en las respuestas, indican que falta información por parte de las empresas encuestadas a la hora de emitir juicios en temas de NIIF. Cabe hacer notar, que se mantiene la tendencia al rededor del $17,5 \%$ que desestima la importancia de las NIIF y menos del 20\% que valora con la máxima puntuación esta importancia.

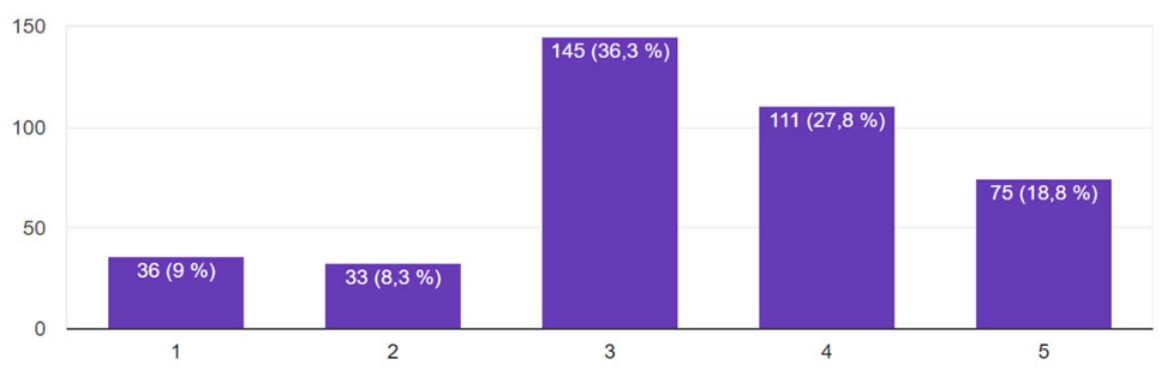

FIGURA 11. Importancia del éxito competitivo de la empresa por implementación de las NIIF

Fuente: elaboración propia.

Con base en lo anterior, es relevante la predisposición que presentan las empresas a la hora de la implementación de las NIIF, se puede evidenciar que los informantes consideran que un $60,9 \%$, no han elaborado un plan estratégico para obtener un proceso exitoso en la implementación y adopción que exigen los estándares internacionales de la información financiera.

Con respecto a la pregunta: "¿Que tanto considera usted, que aspectos fundamentales del área financiera de la empresa, se han visto afectados con la implementación de las NIIF?", en una escala entre 1 (Nada afectados) y 5 (Muy afectados); los encuestados valoraron con un promedio de 3,5, en la distribución se evidencia que la mayor concentración ocurrió en la calificación 3 con un 43\% de los encuestados.

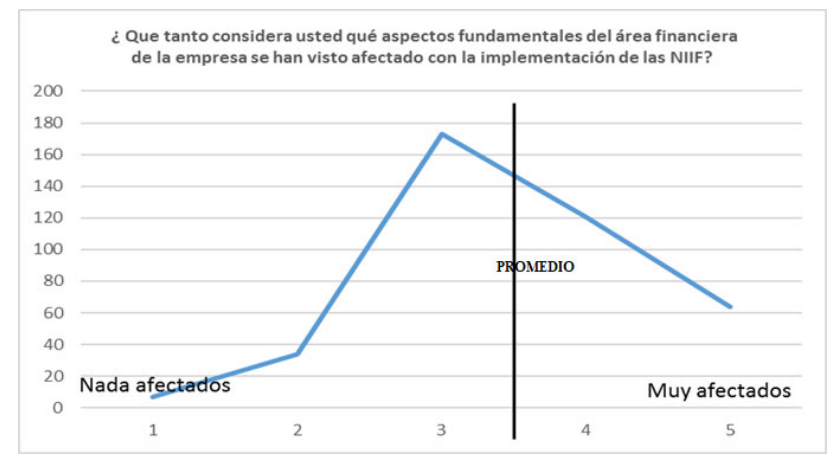

FIGURA 12. Aspectos del área financiera afectados por la implementación de las NIIF

Fuente: elaboración propia.

En este sentido, la calificación que se da a la labor que la empresa ha desarrollado en su proceso sustentable de informes contables basado en las NIIF, es en 62,2\% buena, que contradice las tendencias anteriores en las que se afirma, con un porcentaje similar, que no hay implementación de las NIIF en las empresas y que existe desconocimiento de las normas. Esta contradicción puede deberse a la importancia de las NIIF que nota el encuestado a través del diligenciamiento de la encuesta. 


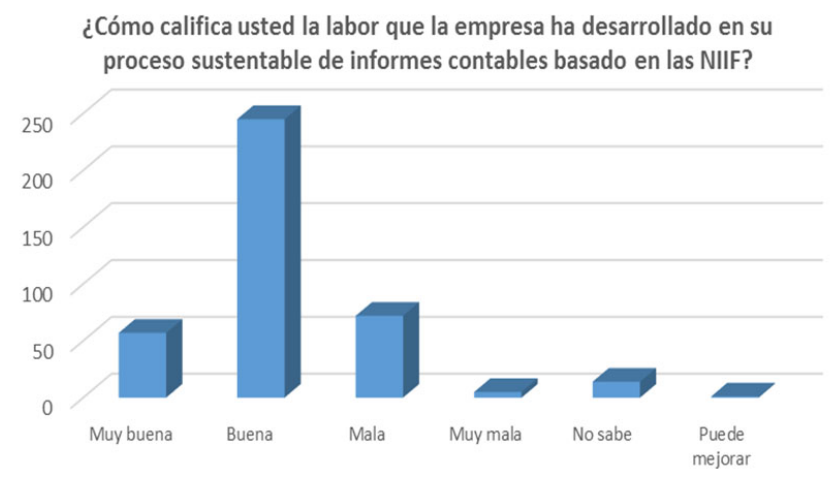

FIGURA 13. Desarrollo del proceso sustentable de informes contables basados en NIIF

Fuente: elaboración propia.

El 55,1\% de las empresas no cuentan con un sistema de auditoría para vigilar el cumplimiento de los requisitos de información continua. Se evidencia la baja gestión organizativa de los temas contables de las empresas que se deben al desconocimiento de los mismos y no a la falta de interés; algunas empresas buscan mantener un estándar, que les permita estar actualizados, pero no cuentan con personal capacitado como se evidenció en el perfil de las personas que respondieron la encuesta.

Un 93\% de los encuestados afirman no conocer empresas que se encuentren en proceso de implementación de las NIIF, y en algunos casos que no saben del tema (esta respuesta se agrupó con no conocen). Con una mayoría que llega al 91,5\%, los encuestados son conscientes de la falta de compromiso a la hora de dar importancia a la implementación de las NIIF en su empresa.

El desconocimiento de las sanciones que tienen lugar por la no implementación de las NIIF, es común para el $84 \%$ de los encuestados; este resultado es consecuente con la tendencia de desconocimiento sobre el tema de NIIF que se ha marcado desde el comienzo de la encuesta.

\section{Conclusiones}

Al aplicar el instrumento de medición se evidencia la baja gestión organizativa en temas contables y financieros que presentan los responsables del proceso de implementación de las NIIF para pymes en las organizaciones, situación que puede ser por falta de conocimiento, capacitación, ayuda de expertos, direccionamiento estratégico, diseño de políticas y desconocimiento en aplicación de sanciones por incumplimiento.

Las pymes del sector industrial de Bogotá no avanzan de manera gradual en el proceso de implementación de las NIIF debido a la dependencia de su conocimiento y recursos, lo que genera retardos e incumplimiento en la presentación de la información. Esto se evidencia en el informe presentado en julio de 2017 por la Superintendencia de Sociedades con corte a diciembre de 2016, donde muestran que más del 50\% cumple con el requisito de enviar la información, pero existen unos porcentajes representativos tanto en envío extraordinario (19\%), como en incumplimiento de esta obligación (16\%); ratificando las conclusiones del estudio de Macías y Quintero (2016).

El presente estudio permite considerar aspectos importantes en el proceso de implementación de las NIIF para pymes del sector industrial en Bogotá. Primero tenemos que esta convergencia genera varios efectos, uno es la falta de compromiso y responsabilidad de la administración en este proceso, hecho que se puede ver reflejado en una resistencia institucional; otro aspecto importante es la falta de capacitación para los responsables de la generación y presentación de la información.

Con respecto a los resultados obtenidos en este estudio se pudo observar que las muestras de las pymes del sector industrial en Bogotá utilizan los recursos entre un 70 y $80 \%$, dejando de aplicar un margen representativo que puede contribuir en incrementar el valor empresarial; resultados que se pueden comparar con los presentados por Abrego et ál. (2016), donde afirma que la calidad del servicio informático favorece los resultados organizacionales. 
Igualmente se puede inferir que falta más consciencia por parte de la administración de las pymes en la inversión de la capacitación del personal, apoyo en expertos, utilización de tecnología y otros recursos; pues esta inversión puede minimizar la dificultad experimentada por las pymes en la implementación de las NIIF. Algunos empresarios de las pymes catalogan la inversión como un gasto y miden los valores a invertir, generando renuencia al cambio e ignorando el valor agregado que esta adopción les puede generar.

Desde luego, la norma establecida para la implementación de las NIIF para pymes, propone la elaboración de un diagnóstico, el diseño de políticas y la elaboración del ESFA; de acuerdo con los resultados de los estudios realizados, se pudo evidenciar que alrededor del $30 \%$ de las pymes encuestadas presentan falencias en este proceso, lo que se ha convertido en un reto bastante difícil, en el que se requiere el acompañamiento de personal experto como lo pueden ser los Contadores Públicos con conocimientos en NIIF.

El análisis de la implementación de las normas internacionales de información financiera NIIF en las pymes del sector industrial de la ciudad de Bogotá llama la atención sobre el conocimiento que tienen los funcionarios del área contable o financiera de estas organizaciones, pero también al personal en su conjunto de estas empresas, los procesos de implementación de las NIIF en las pymes, la elaboración de políticas, la capacitación de los funcionarios sobre las concepciones de las NIIF, la inversión de las pymes en la contratación de personal experto para liderar la implementación de las NIIF, el cumplimento del marco legal y la capacidad de las pymes para brindar información contable y financiera confiable, que les permita incorporarse en la dinámica globalizada de los mercados. Se evidencia la renuencia de los empresarios de las pymes para la adopción de las NIIF, lo que afecta el cronograma establecido por el gobierno nacional, y este mostrando permisividad en lugar de tomar medidas para que su aplicación sea efectiva.

Finalmente, las instituciones reguladoras de las NIIF deberán diseñar políticas más eficientes en el proceso de implementación de las NIIF en las pymes del sector industrial. La superintendencia de sociedades como institución gubernamental en Colombia debe propender por que las pymes incorporen las NIIF, deben buscar aliados estratégicos en el campo académico para acompañar a las pymes en los procesos de capacitación sobre los conceptos de las NIIF y la implementación de la norma.

\section{Referencias}

Abrego, D., Sánchez, Y., \& Medina, J. (2016). Influencia de los sistemas de información en los resultados organizacionales. Recuperado el 11 de mayo de 2016, de http://www.cya.unam.mx/index.php/cya/article/vie $\mathrm{w} / 1049$.

AICPA. American Institute of Certified Public Accountants (2017). Página oficial. Disponible en: http://www.aicp a.org

ANIF. (2016). La gran encuesta pymes. Lectura regional informe de resultados 1er semestre disponible http://anif.co /sites/default/files/encuestas_pyme/2016/09/gep_regional_i-2016.pdf. Recuperado en Mayo de 2017

Bermejo, M., \& Mendoza, Z. (2015). Evaluación de los impactos por la adopción de la NIIF pymes sección 13 del sector comercial. Caso inversiones tropicales. Recuperado el 11 de abril de 2016, de http://190.242.62.234:808 0/jspui/bitstream/11227/2224/1/INFORME\%20FINAL\%202015.pdf

Blanco, V., Quevedo, E., \& Delgado, J. (2009). La estructura financiera de la empresa familiar y el cambio Generacional. Recuperado el 15 de marzo de 2017, de file:///C:/Users/HP/Downloads/ Blanco_Quevedo_Delgado_2009_REFC.pdf

Boz, G., Menéndez, C., Orgaz, N., \& Prior, D. (2015). ¿ Influyen las Normas Internacionales de Información Financiera en el riesgo de las acciones? Recuperado el 24 de septiembre de 2016, de http://www.sciencedirect.com/scienc e/article/pii/S0186104215000145.

Briones, G. (2012). Metodología de la investigación cuantitativa en las ciencias sociales. Bogotá: Instituto Colombiano para el Fomento de la Educación Superior-ICFES. Disponible https://docs.google.com/file/d/0Bwm3dI13n5 jlZmU4OTQwYjgtZWI3Ny00MDY1LWJjMTMtZmU5ZjU1YTI1NGEw/edit?hl=es 
Cámara de comercio de Bogotá. (Noviembre de 2006). Perfil económico y empresarial localidad de Kennedy. Disponible http://bibliotecadigital.ccb.org.co/../6221perfil_economico_kennedy.pdf?sequence=1\&isAllowed $=\mathrm{y}$. Recuperado el 6 de Abril de 2017,

Cañibano, L., \& Gisbert, A. (2007). El proceso de armonización contable internacional, la estrategia europea y la adaptación de la normativa contable en España. Contaduría, Universidad de Antioquia. Recuperado el 30 de julio de 2016, de http://aprendeenlinea.udea.edu.co/revistas/index.php/cont/article/viewFile/2128/1738

Consejo Técnico de la Contaduría Pública (2015) Documento de Orientación Técnica No. 005 Orientación Técnica sobre aplicación de las NIIF para las pymes. Bogotá.

DIAN (2015). Incidencia Fiscal en el Período de Transición de las NIIF, II conversatorio con contadores publicados.

Fito, M., Gómez, F., \& Moya, S. (2010). Efectos del nuevo PGC en los estados financieros: El problema de la comparabilidad de los estados. Recuperado el 19 de mayo de 2016, de http://www.redalyc.org/articulo.oa?id= 43315581008

FMI. Fondo Monetario Internacional (2016). Página oficial. Disponible en: https://www.fmi.org

Fuentes, H., Castiblanco, Y., \& Ramírez, M. (2011). Formulación de las NIF en México: referente de convergencia a NIIF. Recuperado el 17 de julio de 2017 de, http://revistalenguaje.univalle.edu.co/index.php/cuadernosadmin $/ \mathrm{rt} /$ printerFriendly/612/2487.

Galicia, F. (s.f.). Definicion de la pequeña y mediana empresa Scribd., Disponible en https://es.scribd.com/doc/4194 4053/DEFINICION-DE-LA-PEQUENA-EMPRESA-f-f Recuperado el 15 de mayo de 2017

Garza, H., Cortes, K., Méndez, A., \& Rodríguez, M. (2015). Efecto en la calidad de la información ante cambios en la normatividad contable: caso aplicado al sector real Mexicano. Recuperado el 26 de junio de 2017, de http://ww w.sciencedirect.com/science/article/pii/S0186104216300390

González, Á. (2015). Visión sobre los Efectos en la Toma de Decisiones de la implementación de NIF para pymes. Estudio de caso de la Asociación AMORQUIN.

IFRS Foundation (2015). International Financial Reporting Standards. Fundamentos de las Conclusiones del Proyecto de Norma Marco Conceptual para la Información Financiera. Recuperado el 17 de julio de 2017 de, http://ww w.incp.org.co/Site/2015/info/archivos/proyecto-norma-marco-conceptual.pdf

Información mi pymes censo 1990 Y 2005. (2005) disponible https://colaboracion.dnp.gov.co/CDT/Desarrollo\%2 0Empresarial/INFORMACION_MIPYMES_act2012.pdf

Junta de Comercio y Desarrollo (2008). Conferencia de las Naciones Unidas sobre Comercio y Desarrollo. Examén de los problemas de aplicación práctica de las normas internacionales de información financiera. Recuperado el 17 de julio de 2017 de, http://unctad.org/es/Docs/c2isard47_sp.pdf

López, D., Insignaris R., \& Rodríguez, A. (2011). Estrategias de aprendizaje en estudiantes de Educación Media. Revista de la facultad de psicologia de la Universidad Cooperativa de Colombia, 7(13), 130-138. DOI: nd.

Macías, H., \& Quintero, D. (2016). Efectos de la aplicación de IFRS en países menos desarrollados: Revisión de estudios empíricos. Recuperado el 17 de octubre de 2016, de http://revistasum.umanizales.edu.co/ojs/index.p hp/Lumina/article/view/1670.

Martínez, C. (2012). Estadistica y muestreo. Bogotá: Ecoe Ediciones. Disponible https://wwwyyy.files.wordpress.com /2016/06/estadc3adstica-y-muestreo-de-ciro-martc3adnez-b.pdf

Mejía, E., Montes, C., \& Montilla, O. (2008). Fundamentos Teóricos del modelo contable común para las pymes de América Latina: Una alternativa a la regulación contable Internacional IASB. Recuperado el 17 de julio de 2017 de, http://ac.els-cdn.com/S0123592308700378/1-s2.0-S0123592308700378-main.pdf?_tid=35a426c4-6673 -11e7-b8f5-00000aacb362\&acdnat=1499803246_4cc459c6a325f3ed8aa5f088c336ff09

Ministerio de comercio, industria y turismo dirección de Mipymes. (2005) Disponible http://www.mipymes.gov.co/loader.php?IServicio=Documentos\&lFuncion=verPdf\&id=41096\& name $=$ ReporteMipymes03.pdf\&prefijo=file

Ministerio de Comercio, Industria y Turismo. (2012). Definición tamaño empresarial micro, pequeña, mediana o grande. Consulta en línea el 15 de mayo de 2017 de http://www.mincit.gov.co/mipymes/publicaciones/2761/ definicion_tamano_empresarial_micro_pequena_mediana_o_grande 
Muñoz, M. (2016). Impactos en la implementación de las normas internacionales de información financiera (NIIF) en Ferracol S.A.S.

Parra, D. (2016). Análisis de la aplicación de las NIIF en las pymes de la ciudad de cuenca: Percepción, causas e impacto. Recuperado el 24 de diciembre de 2016, de http://dspace.ups.edu.ec/bitstream/123456789/12722/1/UPS-C T006595.pdf.

Presidencia de la Republica. (2004). Ministerio de Comercio Industria y Turismo. Disponible http://www.supertran sporte.gov.co/documentos/ifc/documentos/leyes/Ley\%20905\%20de\%202004.pdf Recuperado el 15 de mayo de 2017

Ramírez H., \& Suarez, L. (2012). Casos sobre NIIF para pymes. Recuperado el 17 de julio de 2017 de, http://es.cal ameo.com/read/00255327470c4aed434b8

Rodríguez, P. (2016). Gestión Financiera en PyMES. Recuperado el 15 de junio de 20117, de file://C:/Users/HP/ Downloads/Dialnet-GestionFinancieraEnPyMES-5833410.pdf

Salas, J. (2015). Impacto financiero de las NIIF en el proceso de implementación en Colombia. Un caso de estudio. COOPACTIVA O.C.

Salazar, E. (2013). Efectos de la implementación de la NIIF para pymes en una mediana empresa ubicada en la ciudad de Bogotá. Recuperado el 16 de mayo de 2016, de file://C:/Users/HP/Downloads/DialnetEfectosDeLaImplementacionDeLaNIIFParaLasPYMESEnUna-5470862.pdf

Secretaria de Desarrollo Económico de Bogotá. (2016). Observatorio de desarrollo económico. Disponible en http://observatorio.desarrolloeconomico.gov.co/base/descargas/public/BoletinEmprendimientoN17febrer o2016.pdf Recuperado el 07 de abril de 2017,

Secretaría Distrital de Planeación - Alcaldía Mayor de Bogotá. Portal SDP > Información Toma Decisiones. Disponible http://www.sdp.gov.co/portal/page/portal/PortalSDP/InformacionTomaDecisiones/Estadisticas /ProyeccionPoblacion:Proyecciones\%20de\%20Poblaci\%F3n

Toro, I., \& Parra, R. (2006). Método y conocimiento: metodología de la investigación, investigación cualitativa, investigación cuantitativa. (pp. 245-254 y 284). Medellín: Fondo Editorial Universidad Eafit.

Vásquez, N. (2013). Impacto de las Normas de Información Financiera en la relevancia de la Información financiera en México. Recuperado el 15 de junio de 2016, de http://www.redalyc.org/articulo.oa?id=39525785004.

Velásquez, L. (2003).Estudio del alcance de la implantación, como apoyo al mejoramiento de los procesos, en las pequeñas y medianas empresas del sector manufacturero en Bogotá. Disponible. http://www.javeriana.edu.co/ biblos/tesis/ingenieria/tesis18.pdf

White, M. (2017). Imperativo de los Estados Unidos: Normas de Contabilidad de alta calidad, aceptadas mundialmente. Recuperado el 24 de julio de 2017 de http://www.nicniif.org/home/novedades/imperativo-delos-estados-unidos-normas-de-contabilidad-de-alta-calidad-aceptadas-mundialmente.html

Wong, A. (2007). Convergencia y armonización de la normativa contable: hacia la contabilidad internacional. Contabilidad y Negocios: Revista del Departamento Académico de Ciencias Administrativas, 2(4), 7-15. DOI: nd.

\section{Notas}

* Artículo de investigación científica y tecnológica.

\section{Licencia Creative Commons CC BY 4.0}

Para citar este artículo: Orobio, A., Rodríguez, E. M., Acosta, J. C. (2018). Análisis del impacto de implementación de las normas internacionales de información financiera (NIIF) en el sector industrial de Bogotá para PYMEs. Cuadernos de Contabilidad, 19(48), 1-18. https://doi.org/10.11144/Javeriana.cc19 -48.aiin 